\title{
AURIKULOMEDIZINISCHE OPTIONEN BEI SARS-COV-2, SPEZIELL LONG-COVID-19
}

\section{Therapeutic Options from the Perspective of Auriculomedicine in SARS-Cov-2, especially Long-COVID-19}

\section{Zusammenfassung}

Nach mehr als einem Jahr Corona-Pandemie zeigt sich, dass ein nicht unerheblicher Prozentsatz der Erkrankten an anhaltenden Symptomen leidet. Die Ursachen sind nur teilweise geklärt. Aufgrund der großteils funktionalen Natur der Beschwerden bietet sich die Ohrakupunktur/ Aurikulomedizin als Behandlungsoption an. Es wird ein Procedere vorgeschlagen, das sich hauptsächlich vom Konzept der Linie der Infekte ableitet. ZurVerbesserung des inflammatorischen Status scheint die Ernährung eine wichtige Rolle zu spielen. MitVitamin D und Astaxanthin werden zudem zwei Nahrungsergänzungsmittel in das Behandlungskonzept integriert. Im Sinne der Prophylaxe wird ein Vorgehen mit Implantat-Akupunktur diskutiert. Weiterhin wird ein mögliches Procedere beim akuten COVID vorgestellt, welches auf aurikulomedizinischen Erkenntnissen beruht.

\section{Schlüsselwörter}

Long-COVID, Post-COVID, SARS-CoV-2, Ohrakupunktur, Aurikulomedizin, Linie der Infekte, Ernährung

\section{Summary}

After more than a year of corona pandemic, it is apparent that a considerable percentage of those affected suffer from persistent symptoms. The causes are only partially understood. Due to the largely functional nature of the symptoms, ear acupuncture/auriculomedicine offers an interesting treatment option. A procedure mainly derived from the concept of the line of infections is proposed. To improve the inflammatory status, nutrition seems to play an important role. With Vitamin D and Astaxanthine, two dietary supplements are also integrated into the treatment concept. In the sense of a prophylaxis against COVID-19, a procedure with implant acupuncture is discussed. Furthermore, a possible proceeding for acute COVID-19, based on auriculomedical findings, is presented.

\section{Keywords}

long COVID, post COVID, SARS-CoV-2, ear acupuncture, auriculomedicine, line of infections, nutrition

\section{Therapie des Long-COVID-19}

Die SARS-CoV-2-Pandemie dauert nun schon über 1 Jahr. Zunehmend sind wir in der Praxis mit Langzeitfolgen von durchgemachten Erkrankungen konfrontiert. Die Zahlen zur Prävalenz schwanken stark und hängen teilweise von der Schwere des durchgemachten akuten Infekts ab. Es ist jedoch auffallig, dass auch Patienten mit mildem Verlauf Langzeitsymptome entwickeln können, wenn auch seltener [1].

\section{Definition Long-COVID-19}

Die vorgeschlagenen Definitionen sind bis anhin nicht einheitlich. Das britische National Institute for Health and Care Excellence (NICE) beschreibt unter „Long-COVID“ Symptome, die über 4 Wochen nach Krankheitsbeginn persistieren [2]. Gemäß NICE kann der Begriff LongCOVID zusätzlich unterteilt werden in ,,anhaltendes symptomatisches COVID-19“ für Symptome von 4-12 Wochen und das „Post-COVID-19“ für Symptome, die mehr als 12 Wochen anhalten. 


\section{Krankheitsbild}

COVID-19 ist eine komplexe Krankheit. Sicher ist die Lunge das hauptsächlich betroffene Organ und hat daher eine dominierende Stellung im Krankheitsgeschehen. Es ist jedoch unbestritten, dass COVID-19 eine systemische Erkrankung ist. So ließ sich in einer autoptischen mikrobiologischen und histologischen Studie dasVirus in vielen Organen nachweisen. Interessanterweise zeigten sich aber strukturelle Schäden fast nur im Lungengewebe, wo die Viruslast am höchsten ist [3].

Der Schweregrad einer COVID-19-Erkrankung ist sehr variabel. Das Spektrum reicht von asymptomatischen Infektionen bis zu schwersten, hochseptischen Verläufen. Trotz des sehr unterschiedlichen Schweregrads der akuten Erkrankung ist das grundsätzliche Muster der Langzeit-

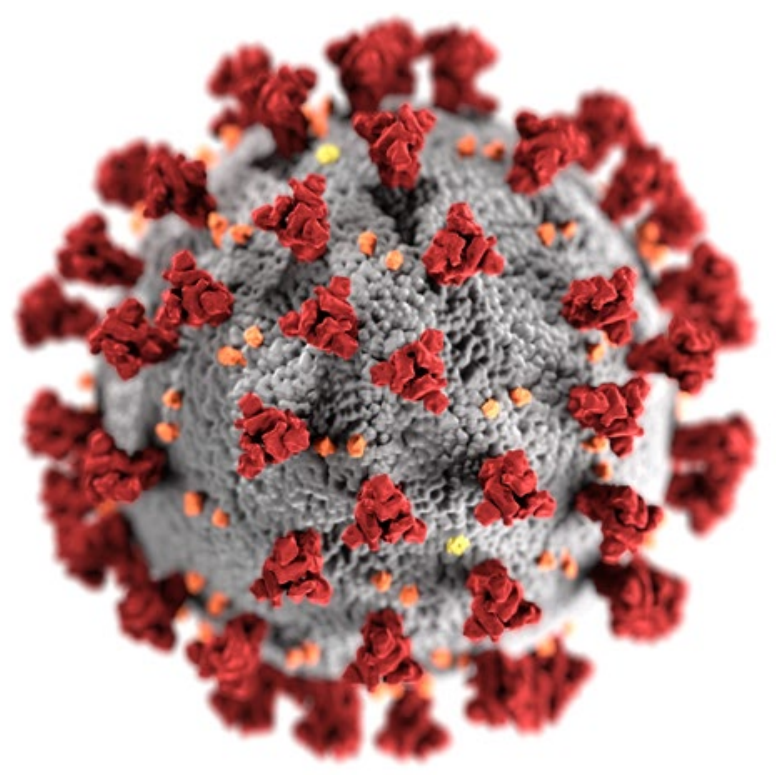

Abb. 1: Das SARS-CoV-2-Virus mit den prominenten Spike-Proteinen. Von CDC/Alissa Eckert, MS

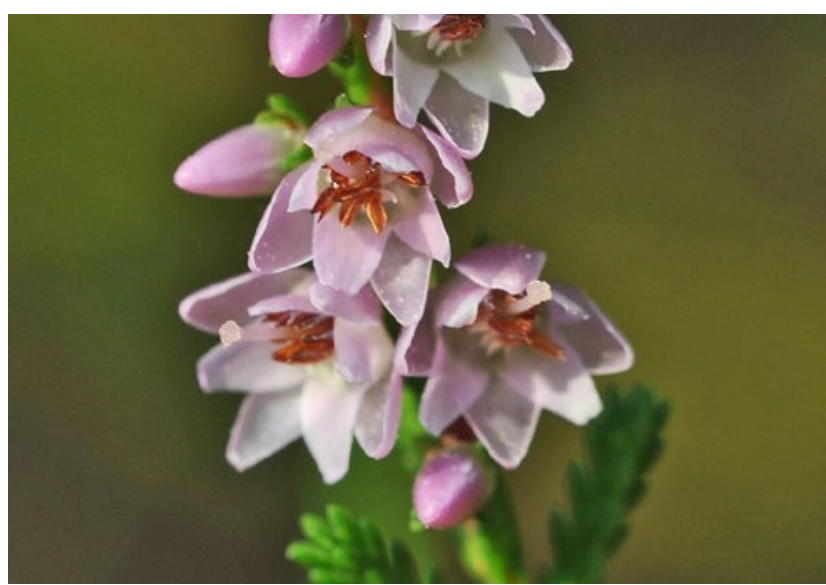

Abb. 2: Bachblüte Heather, (CZeynel Cebeci-https://commons. wikimedia. $\mathrm{org} / \mathrm{w} / \mathrm{index}$.php?curid $=34719041$ folgen ähnlich. Folgende sind die häufigsten und relevantesten Beschwerden [4]:

- Müdigkeit,

- Depression,

- Dyspnoe,

- Kopfschmerzen,

- olfaktorische Ausfälle,

- kognitive Beeinträchtigungen.

\section{Pathogenese des Long-COVID-19}

Als Ursache des Long-COVID-19 werden verschiedene Faktoren diskutiert. Bei schweren COVID-19-Verläufen können natürlich strukturelle Schäden im Lungengewebe eine Rolle spielen. Dazu kommt die psychische Belastung, welche die Erkrankung mit sich bringt, insbesondere bei beatmeten Patienten. Bei milden COVID-19-Verläufen greift diese Erklärung jedoch nicht. Hierzu wird eine Viruspersistenz diskutiert, welche durch eine ungenügende, respektive inadäquate Immunreaktion bedingt ist [5]: Einerseits kann dies durch eine hyperge, also zu schwache Immunlage bedingt sein. Andererseits kann sich auch eine hypererge Immunlage bilden, welche durch sogenannte Super-Antigene unterhalten wird: Dies führt in der Folge wiederum zur Schwächung der eigentlichenVirusabwehr und gleichzeitig zu den gefürchteten entzündlichen Gewebeschäden.

\section{Therapie des Long-COVID-19}

Die schulmedizinischen Möglichkeiten der Therapie beschränken sich im Wesentlichen auf symptomatische Maßnahmen wie die Behandlung von Dyspnoe, kardialen Problemen Schlafstörungen, Depressionen usw. Zudem werden psychotherapeutische Begleitung sowie Instruktion von Coping-Strategien angeboten.

Aufgrund des großteils funktionalen Charakters des Long-COVID-19 drängt sich ein komplementärmedizinisches Vorgehen geradezu auf. Insbesondere die RACkontrollierte Ohrakupunktur bietet uns einen sehr zielgerichteten Ansatz. Das im Folgenden beschriebene Vorgehen fußt auf der Störfelder-basierten Ohrakupunktur nach Bahr [6] und den Erfahrungen mit der Erreger-spezifischen Immunstimulation über die Linie der Infekte $[7,8,9]$.

\section{Der aurikulomedizinische Zugang zum Long- COVID-19}

Zur Evaluation der relevanten Punkte ist es sehr hilfreich, eine krankheitsspezifische Information zu haben, womit man mittels RAC das Ohr absuchen kann. Wie in der Bahr'schen Schule oft verwendet, können wir dies mittels 


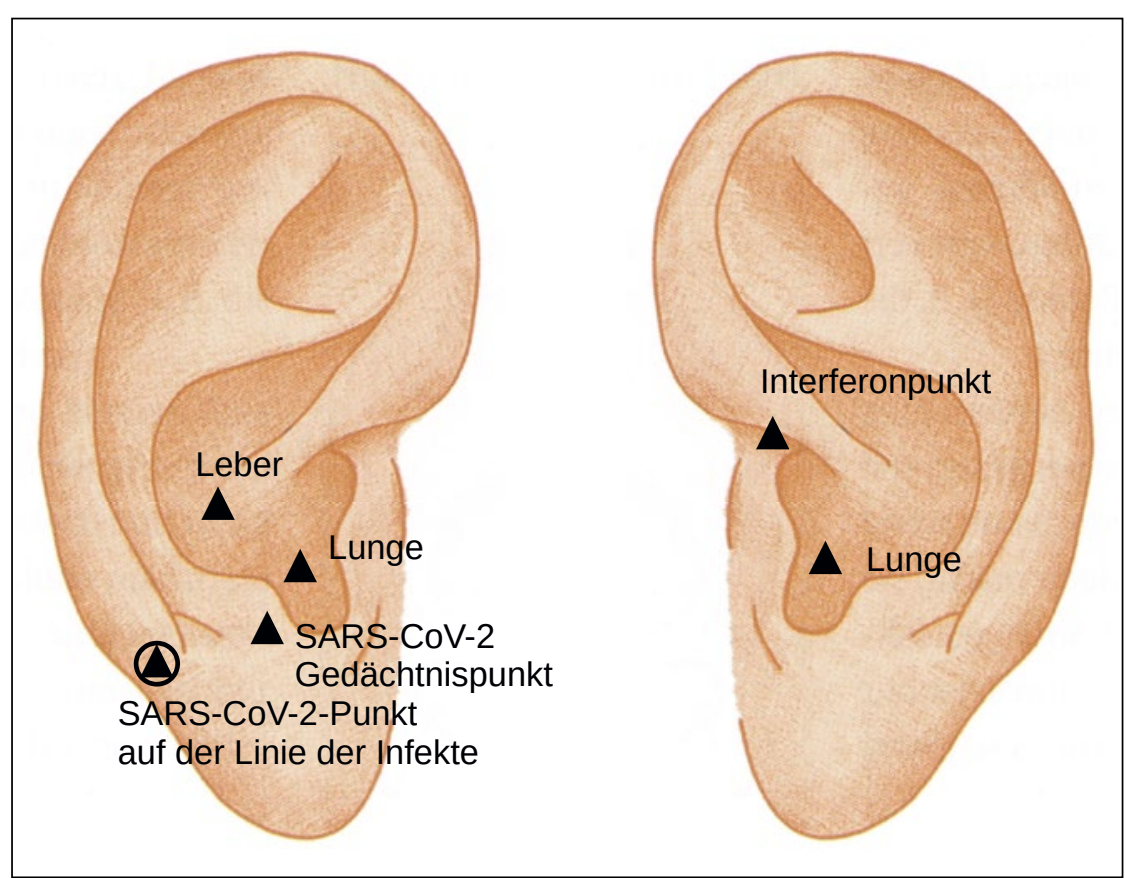

Abb. 3: Punkteschema zur Therapie des Long-COVID

biologisch spezifisch wirkender Frequenzen bewerkstelligen. Im Falle des SARS-CoV-2 (Abb. 1) konnte dafür die Frequenz 6282.6 Hz eruiert werden. Diese Frequenz kann mit einem geeigneten Laser oder dem Frequenzhämmerchen hergestellt werden. Steht diese Möglichkeit nicht zurVerfügung, kann alternativ mit einer Ampulle der Bachblüte Heather gesucht werden (Abb. 2).

Damit werden nun mithilfe des RAC die Ohrmuscheln abgesucht. Die im Folgenden beschriebenen Punkte zeigten sich als durchgehendes Muster bei fast allen betroffenen Patienten. Der erfahrene Aurikulomediziner kann mit dieser Frequenz im Einzelfall auch noch weitere aktive Punkte finden, die ins Behandlungsschema inte-

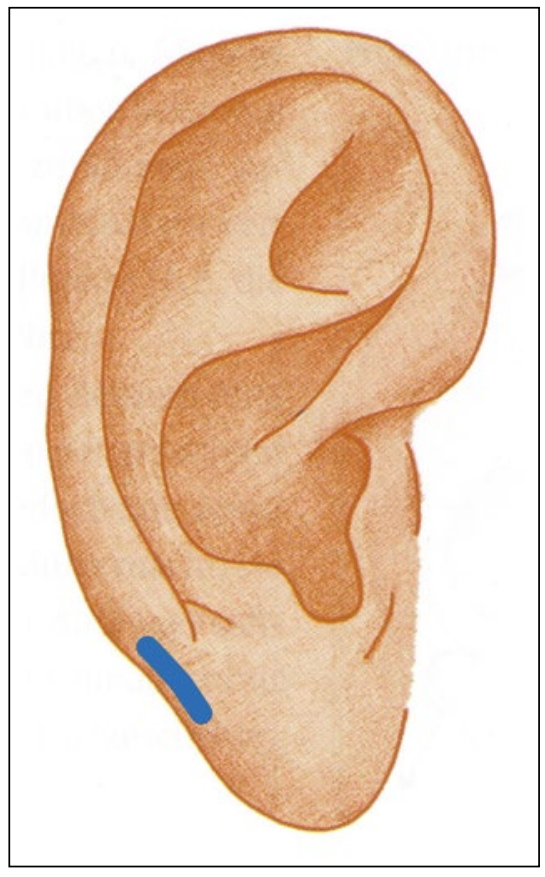

Abb. 5: Der Viren-Abschnitt auf der Linie der Infekte griert werden können. Mit dem mit 6282.6 Hz modulierten Laser wird entweder direkt am Ohr mit diagnostischer Intensität gesucht oder diese Frequenz wird mit voller Leistung peripher aufgelegt und die Ohren werden mit dem Hybridhämmerchen nach Bahr abgesucht. Wird der RAC noch nicht beherrscht, können die im Folgenden genannten Punkte natürlich auch mit dem Punktsuchgerät geprüft werden (Abb. 3).

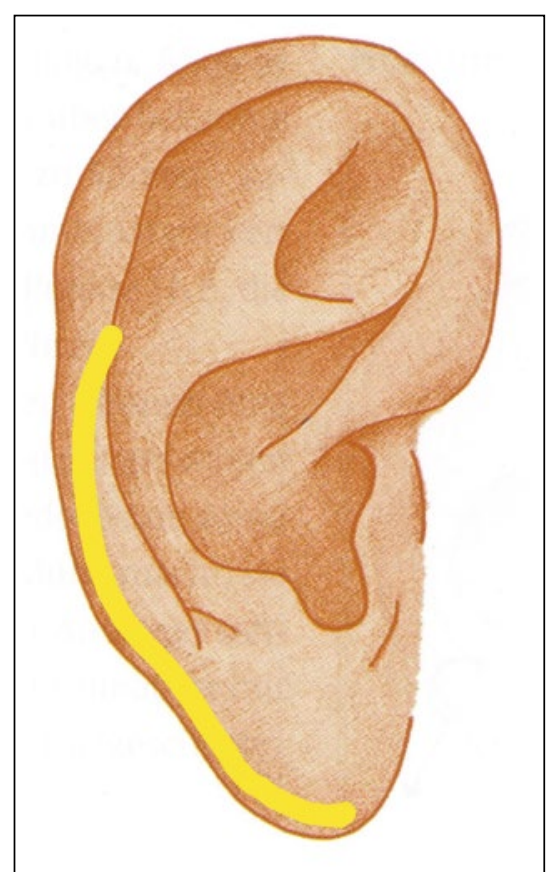

Abb. 4: Die Linie der Infekte

\section{SARS-CoV-2 und die Linie der Infekte}

Die Behandlung von chronisch-persistierenden Infekten lässt sich elegant über die Linie der Infekte behandeln (Abb. 4).

Bei chronisch-persistierenden Infektionen, wie EBV, Borreliose und Lamblien, lässt sich damit eine erregerspezifische Immunstimulation erzielen. Dies hat sich in den letzten 12 Jahren in der Praxis immer wieder bestätigt. Dazu müssen die mittels RAC gefundenen aktiven Punkte auf der Linie der Infekte langzeitig akupunktiert werden [7,8,9]. Die Punkte der Viren finden sich in einem kleinen Abschnitt, ungefähr auf der Höhe des Hirnstamms (Abb. 5).

Die Langzeit-Punktstimulation wird sinnvollerweise mit Templax-Implantaten gemacht (Abb. 6) Auf Long-COVID-19 bezogen wird der entsprechende aktive Punkt vorzugsweise mittels Templax rapid stimuliert. Alternativ kann auch eine 2- bis 4-malige Applikation einer ASP-Dauernadel verwendet werden. Dieser Punkt auf der Linie der Infekte muss so lange akupunktiert werden, bis er in den Nachkontrollen nicht mehr aktiv ist. Kommen bei einem Patienten Nadeln 


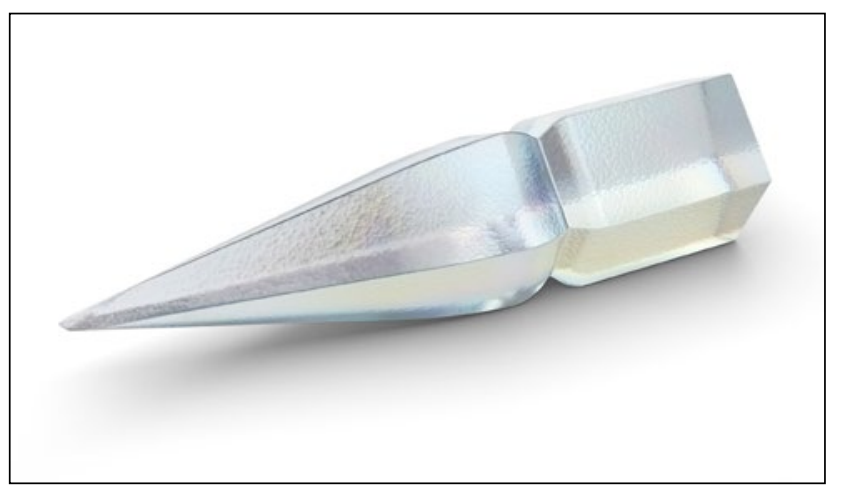

Abb. 6:Templax-Implantat

nicht infrage, oder ist man sich bei der Punktdiagnostik nicht sicher, kann mit gewissen Abstrichen auch die Bachblüte Heather verschrieben werden. Die Wirkung ist dezenter als die Akupunktur, jedoch ausreichend. Beim Erwachsenen werden 3 Tropfen über den Tag verteilt eingenommen.

\section{Störfelder und weitere Punkte}

Werden die Störfelddiagnostik und -therapie nach Bahr beherrscht, sollten diese zwingend gemacht werden [6]. Erfahrungsgemäß zeigen sich fast immer starke Störfelder im Bereich der Lunge und dies meist beidseitig. Zusätzlich sollte auch immer der Organpunkt der Leber getestet werden. Weitere Punkte, die gesucht werden müssen, sind der Interferon-Punkt nach Bahr auf der nicht dominanten Seite sowie ein zentraler Gedächtnis-Punkt auf der dominanten Seite. Dieser befindet sich etwa $2 \mathrm{~mm}$ unterhalb des Gonadotropin-Punkts auf der dominanten Seite und imponiert als Silber-Punkt. Die gefundenen Punkte werden mit Gold-, respektive Silbernadeln oder durchaus auch direkt mittels ASP-Dauernadeln behandelt.

\section{Ernährung und Nahrungsergänzungsmittel}

Es spricht vieles dafür, dass Long-COVID-19 mit einer persistierenden Inflammation im Zusammenhang steht. Deshalb sollte auf eine antiinflammatorische Ernährung geachtet werden [10]. Dies lässt sich mit einer vollwertigen, pflanzenbetonten Ernährung erreichen, die reich an $\mathrm{Ge}-$ müse, Früchten, Beeren, Vollkorn und Nüssen ist. Die Ernährungspyramide der Deutschen Gesellschaft für Ernährung ist ein praktikabler Ansatz [11]. Aus TCM-energetischen Überlegungen sollte vor allem warm gegessen werden. Rohkost sollte bei Krankheit weitgehend vermieden werden.

Es gibt gute Gründe, generell zurückhaltend mit der unkritischen Empfehlung von Nahrungsergänzungsmitteln zu sein. Es gibt aber einige interessante Kandidaten beim akuten COVID wie auch beim Long-COVID-19. Zwei haben sich aus meiner Sicht klinisch und gemäß RACDiagnostik besonders bewährt:

1. Es gibt eine Vielzahl von Studien, die tiefere Spiegel von 25-OH-Vitamin D mit einem schwereren COVID-19-Verlauf assoziieren. Zudem gibt es einige kleinere Interventionsstudien mit eingeschränkter Aussagekraft, die den Zusammenhang stützen. Die Evidenz wird immer besser, aber die Diskussion bleibt nach wie vor kontrovers [12]. Beim Long-COVID-19 macht ein rasches Beheben eines 25-OH-Vitamin-D-Mangels mit Zielwert von mindestens $75 \mathrm{nmol} / 1$ (30 ng/ml) Sinn. Der optimale Spiegel liegt wahrscheinlich bei 100-150 $\mathrm{nmol} / 1$ (40-60 ng/ml). Es empfiehlt sich eine rasche Aufdosierung (s.u. Kasten), da das Erreichen des Zielspiegels sonst Monate dauern kann. Die Erhaltungsdosis zum Erreichen dieses Spiegels liegt meist zwischen 2000 und 4000 IE pro Tag und sollte im Labor kontrolliert werden.

2. Astaxanthin, ein Carotinoid, ist ein potentes lipophiles Antioxidans. Es besteht eine hohe Evidenz für umfassende antiinflammatorische Effekte [13]. Interessant ist die Substanz auch aus Sicht der Aurikulomedizin. Einerseits zeigt die Testung mit dem RAC einen expliziten Bezug zum Punkt Lunge 7, dem Organpunkt Lunge in der Ohr-Somatotopie. Die Lunge ist bei COVID-19 das in der Regel am stärksten betroffene Organ. Dessen Yin-Qi wird außerordentlich geschwächt und braucht intensive Unterstützung. Astaxanthin stärkt sehr effektiv das Lungen-Yin-Qi. Zusätzlich zeigt sich, dass nach Astaxanthin-Einnahme der Punkt auf der Linie der Infekte praktisch gelöscht wird, respektive kaum mehr nachweisbar ist. Das sind aus aurikulomedizinischer Sicht zwei starke Gründe für dessen Einsatz sowohl beim akuten COVID-19 als auch beim LongCOVID-19. Die Dosierungsempfehlung beim LongCOVID-19 ist $4 \mathrm{mg}$ pro Tag. Die Einnahme sollte gemäß TCM zur Lungenzeit erfolgen, also idealerweise zum Frühstück.

Formel zur Berechnung der Initial- und Erhaltungsdosis von Vitamin D

Aufdosierung (Gabe wird über 10 Tage verteilt):

$40 \mathrm{x}$ (Zielwert in $\mathrm{nmol} / \mathrm{l}$ - Ausgangwert in $\mathrm{nmol} / \mathrm{l}$ ) $\mathrm{x}$ Körpergewicht in $\mathrm{kg}$

Erhaltungsdosis: 2000-4000 IE pro Tag, Kontrollen im Labor 


\section{Fallbeispiel}

56-jähriger Patient mit leichterem COVID im Oktober 2020. Aus der Vorgeschichte ist ein Burnout 2017 zu erwähnen. Außer einer leichten Adipositas bestanden keine weiteren Risikofaktoren für schweres COVID-19. Zwei Monate nach Abklingen der akuten Erkrankung persistierten eine bleierne Müdigkeit, Muskelschmerzen und ein thorakales Oppressionsgefühl mit Kurzatmigkeit. Aufgrund der Belastungsintoleranz und Konzentrationsschwäche wurde er arbeitsunfähig geschrieben. Die RAC-Diagnostik ergab Folgendes: In der Voruntersuchung nach Bahr fand sich gleichzeitig eine Inversion im Bereich der rechten Lunge sowie eine Oszillation am Leberpunkt. Die beiden Punkte reagierten eindeutig auf die Coronafrequenz von $6282.6 \mathrm{~Hz}$. Der Zusammenhang dieser beiden starken Störfelder mit COVID-19 konnte durch die Tatsache erhärtet werden, dass die periphere Auflage von Astaxanthin und der Bachblüte Heather sowohl Oszillation wie auch Inversion kurzzeitig zum Verschwinden brachten. Mit der Coronafrequenz mit diagnostischer Intensität fanden sich nebst dem Coronapunkt auf der Linie der Infekte zudem folgende aktiven Punkte: Lunge links und Interferonpunkt links. Therapeutisch behandelte ich den Coronapunkt auf der Linie der Infekte mit einerTemplax rapid. Die weiteren genannten Punkte wurden mit ASP-Dauernadeln versehen. Zusätzlich akupunktierte ich den Silber-Blockadepunkt nach Bahr präaurikulär in Silber [9]. Aufgrund des relativ tiefen 25-OH-Vitamin-DSpiegels von $52 \mathrm{nmol} / 1$ verordnete ich ihm zur Aufdosierung 30.000 IE pro Tag über 8 Tage, zudem Astaxanthin $4 \mathrm{mg}$ morgens. Jeweils nach Herausfallen der ASP-Dauernadeln wurde der Patient erneut mit ASP-Dauernadeln akupunktiert. Anlässlich der dritten Akupunktur, 6 Wochen nach der Erstbehandlung, waren die meisten Symptome verschwunden, der Patient ist wieder voll arbeitsfähig. Es persistierte zu dem Zeitpunkt noch eine leichte Belastungsintoleranz sowie eine diskrete Kurzatmigkeit bei schnellem Treppensteigen.

\section{COVID-19-Prophylaxe?}

Können wir nicht nur Infektionen und Folgen von Infektionen behandeln, sondern auch schon präventiv wirken? In der Komplementärmedizin, insbesondere der traditionellen chinesischen Medizin, ist das Konzept der Grippeund Erkältungsprävention verbreitet. China hat in den letzten Jahrzehnten einige Seuchen durchgemacht. Das Land hat deshalb viele Erfahrungen mit deren Therapie mittels traditioneller chinesischer Medizin gemacht. Dies gilt auch für die Prävention. Ein Review gibt einen Über- blick zurTherapie und Prävention bezüglich SARS-CoV-2 [15].

Viele Patienten wollen sich aus verschiedenen Gründen nicht gegen SARS-CoV-2 impfen lassen. Es stellt sich hier die Frage, inwiefern wir diese Patienten auch präventiv mit den Möglichkeiten der Ohrakupunktur unterstützen können. Dazu kommt, dass die Impfung keinen vollständigen Schutz vor COVID-19 bietet, was sich durch die natürliche Tendenz des Virus zur Mutation verstärkt. Zudem ist noch völlig unklar, wie lange der Impfschutz anhalten wird. Ich möchte hier das Vorgehen kurz vorstellen, welches wir im Akupunkturzentrum Biel seit Mai 2020 anwenden. Dies leitet sich von den guten Erfahrungen bei der Therapie des Herpes simplex ab.

\section{Als Beispiel die Therapie des Herpes simplex}

Beim rezidivierendem Herpes simplex konnten wir in den letzten Jahren gute Erfahrungen mit dessen Prophylaxe machen. Dabei wird mit der geeigneten Technik der Entsprechungspunkt dieses Virus auf der Linie der Infekte Langzeit-akupunktiert. Dieser befindet sich auf dem Unterabschnitt der Linie der Infekte, im Bereich derViren (s. Abb. 5). Um den Punkt genau zu lokalisieren, braucht es die Herpes-spezifische Frequenz, welche mit dem Laser oder dem Frequenzhämmerchen peripher aufgelegt wird. Es handelt sich um die $2735.2 \mathrm{~Hz}$. Die Dauerstimulation des Punktes geschieht mit Templax-Implantaten, meist mit der Variante supra, welche eine Wirkdauer von ca. 3 Jahren ermöglicht. Damit lässt sich die Rezidivrate massiv reduzieren. Kommt es trotzdem zu einem Ausbruch, ist er meist schwach. Aufgrund des Leidensdrucks ist diese Therapie vor allem beim Herpes genitalis hilfreich, funktioniert jedoch identisch auch beim Herpes labialis. Da die Herpes-Viren naturgemäß nicht eradizierbar sind, kommt es nach der Auflösung des Implantats in aller Regel wieder zu einer Häufung der Eruptionen. Deshalb muss das Templax-Implantat nach seiner Auflösung erneut gesetzt werden.

\section{Übertragung auf SARS-CoV-2}

Ausgehend von diesen Erfahrungen haben wir das Vorgehen auf SARS-CoV-2-Prophylaxe übertragen. Der Punkt SARS-CoV-2 auf derVirenlinie ist naturgemäß beim noch nicht durchgemachten Infekt nicht aktiv. Wir müssen also den Punkt diagnostisch darstellbar machen. Dazu wird die SARS-CoV-2-Frequenz $6282.6 \mathrm{~Hz}$ mit dem Laser oder dem Frequenzhämmerchen peripher aufgelegt. DerVirenPunkt kann mit der Silber-Seite des Hybridhämmerchens nach Bahr gefunden werden. Dieser Punkt wird dann ana- 
log zum Vorgehen bei Herpes simplex mittels LangzeitImplantat (,Primo“ oder sogar „Supra“) versehen.

Hat man die technischen Möglichkeiten nicht, sei es wegen fehlenden Lasers oder weil der RAC noch nicht beherrscht wird, kann alternativ auf die Dauereinnahme der Bachblüte Heather zurückgegriffen werden. Die Dosierung für Erwachsene ist je 1 Tropfen morgens und abends.

Zusätzlich zur Akupunktur empfiehlt sich die Einnahme von $4 \mathrm{mg}$ Astaxanthin morgens und eine Vitamin-DSupplementation mit Zielwert 100-150 nmol/1 (40-60 $\mathrm{ng} / \mathrm{ml})$.

Mit einer vollwertigen, pflanzenbetonten Ernährung und regelmäßiger Bewegung können die 3 hauptsächlichen Risikofaktoren für ein schweres COVID-19 günstig beeinflusst werden: Adipositas, arterielle Hypertonie und Diabetes. Zudem kann damit der antioxidative Status und die chronische Inflammation verbessert werden.

\section{Möglichkeiten beim akuten Infekt}

Die Akupunktur verbietet sich hier aus offensichtlichen Gründen. Eine zur Akupunktur des SARS-CoV-2-Punktes analoge Stimulation kann jedoch mit der Einnahme der Bachblüte Heather gemacht werden. Wie beim Vorgehen bei Long-COVID werden 3 Tropfen über den Tag verteilt eingenommen.

Auch Astaxanthin sollte so rasch als möglich verabreicht werden. Die Dosierung beträgt $3 \times 4 \mathrm{mg}$ pro Tag.

Inwiefern Vitamin D beim akuten Infekt einen positiven Effekt auf denVerlauf hat, ist noch nicht eindeutig klar. Es gibt jedoch eine gewisse Evidenz für einen günstigeren Verlauf der Schwere der Krankheit. Bei fehlender Kontraindikation, wie Hyperkalzämie, Hyperparathyreoidismus und Sarkoidose, können gefahrlos 4000 IEVitamin D3 pro Tag verordnet werden [14].

Der Vollständigkeit halber erwähne ich weitere Substanzen, deren Effekt auf COVID-19 diskutiert wird: Zink, Vitamin C, Melatonin,Vitamin K2.

\section{Fallbeispiel}

Ein 15-jähriger autistischer Junge infizierte sich im Dezember 2020 in der Pflegeeinrichtung mit SARS-CoV-2.

\section{Literatur}

[1] Halpin S, McIvor C, Whyatt G et al.: Postdischarge symptoms and rehabilitation needs in survivors of COVID-19 infection: A cross-sectional evaluation.J MedVirol 2021; 93: 1013-1022.
Er entwickelte Fieber bis $39^{\circ}$, einen starken trockenen Husten und war gemäß der betreuenden Mutter muskulär hypoton und apathisch. Die Atemfrequenz betrug zwischen 24 und 30 pro Minute.Aufgrund des Autismus wäre eine Hospitalisation sehr problematisch gewesen. Der Patient, wie auch die betreuende Mutter, sind schwarzer Hautfarbe. Deshalb und aufgrund der Winterzeit konnte bei beiden von einem Vitamin-D-Mangel ausgegangen werden. Bei fehlender Anamnese bezüglich CalciumStoffwechselstörung verordnete ich deshalb 20.000 IE Vitamin D3, dreimal pro Tag während 3 Tagen, also total 180.000 IE zur raschen Aufdosierung, zudem Astaxanthin $4 \mathrm{mg}$, dreimal pro Tag. Die Bachblüte Heather war leider in der lokalen Apotheke nicht erhältlich. Nach einem Tag der Einnahme war der Patient gemäß Aussagen der Mutter deutlich wacher. Bei persistierendem Husten ging die Atemfrequenz auf 20 pro Minute zurück. Der Patient hatte innert 4 Tagen entfiebert, wobei sich auch der Husten gelegt hat.

Die Mutter war durch die Pflege auch einem hohen Infektionsrisiko ausgesetzt. Deshalb habe ich ihr prophylaktisch Vitamin D3 mit einer Gesamtdosis von 240.000 IE über 4 Tage verordnet, wie auch Astaxanthin $4 \mathrm{mg}$, dreimal pro Tag. Sie hatte keine COVID-Symptomatik entwickelt. Einen Labortest mit PCR und später einen serologischen Antikörpertest ließ sie sich nicht machen. Sie wurde eindringlich auf die Isolations- und Quarantäne-Maßnahmen hingewiesen.

\section{Fazit}

Währenddem die klassische Schulmedizin einen sehrVirenzentrierten Fokus hat, hat die Komplementärmedizin besonders das „Terrain“, resp. die Immunlage, im Blick. Man könnte es ganz vereinfacht umschreiben:Wie bringt man den Organismus in die Lage, dass er besser mit dem Virus umgehen kann? Das beschriebene aurikulomedizinische Vorgehen hat sich in der Praxis, insbesondere bei derTherapie des Long-COVID-19 bewährt.

Es bestehen keine Interessenkonflikte.

[2] National Institute for Health and Care Excellence. COVID-19 rapid guideline: managing the long-term effects of $\mathrm{CO}$ VID-19. NICE guideline [NG188]. Published 18 December 
2020. https://www.nice.org.uk/guidance/ng188. Stand: 17.05.2021.

[3] Deinhardt-Emmer S, Wittschieber D, Sanft J et al.: Early postmortem mapping of SARS-CoV-2 RNA in patients with COVID-19 and the correlation with tissue damage. Elife 2021; 10: e60361.

[4] Sudre CH, Murray B, Varsavsky T et al.: Attributes and predictors of Long-COVID: analysis of COVID cases and their symptoms collected by the Covid Symptoms Study App. medRxiv Oct 21, 2020. doi: https://doi.org/10.1101/2020.10. 19.20214494 .

[5] Jacobs JJL: Persistent SARS-2 infections contribute to long COVID-19. Med Hypotheses 2021; 149: 110538.

[6] Bahr F, Strittmatter B: Das große Buch der Ohrakupunktur. Stuttgart: Hippokrates; 2010.

[7] Scholtes C:Therapie chronischer Erkrankungen - Die Linie der Infekte. Schmerz \& Akupunktur 2011; 1: 5-9.

[8] Scholtes C: Die Linie der Infekte und das Metagenom - Integration in das System der Gedächtnispunkte. Akupunkt Aurikulomed 2015; 41: 23-29.

[9] Scholtes C: Die Emotion hinter der Infektion - Fortgeschrittene aurikulomedizinische Techniken zur Behandlung von chronischen Infektionen. Akupunkt Aurikulomed 2019; 45: 16-21.

[10] Eiser A: Could Dietary Factors Reduce COVID-19 Mortality Rates? Moderating the Inflammatory State.J Altern Complement Med 2021; 27: 176-178.

[11] Deutsche Gesellschaft für Ernährung e.V.:Vollwertig essen und trinken nach den 10 Regeln der DGE. https://www.dge.de/ index.php?id=52. Stand: 17.05.2021.

[12] The Lancet Diabetes Endocrinology. Editorial:Vitamin D and COVID-19: why the controversy? Lancet Diabetes Endocrinol 2021; 9: 53.

[13] Fakhri S, Nouri Z, Zachariah Moradi S et al.: Astaxanthin, COVID-19 and immune response: Focus on oxidative stress, apoptosis and autophagy. Phytother Res 2020;34:2790-2792.

[14] Ross AC, Manson JE, Abrams SA et al.: The 2011 report on dietary reference intakes for calcium and vitamin $\mathrm{D}$ from the Institute of Medicine: what clinicians need to know. J Clin Endocrinol Metab 2011; 96: 53-58.

[15] Zhenyu $Z$ et al.: Prevention and treatment of COVID-19 using Traditional Chinese Medicine:A review. Phytomedicine 2021; 85: 153308 .

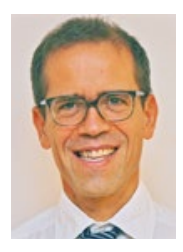

Dr. med. Christoph Scholtes

Bahnhofstraße 16

CH-2502 Biel

scholtes@akuzen.ch

\section{Qualität E Tradition seit I950}

\section{Laser wirkt! Die sanfte Art zu heilen. Schmerzfrei, wirksam, effektiv $\mathcal{E}^{\circ}$ wirtschaftlich.}

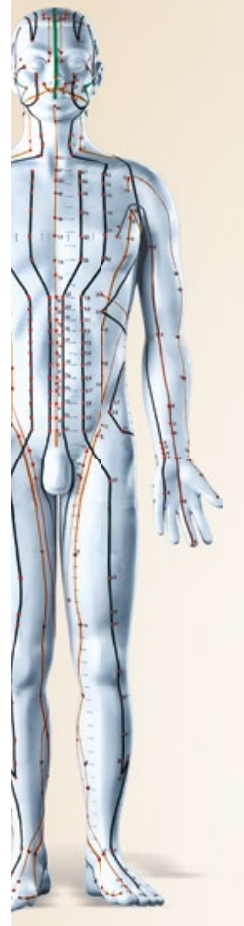

Therapielaser KI. 3B und KI. 4 für Ohrund Körperakupunktur, lokale Therapie.

\section{Akupunkturbedarf Karl Blum GmbH} Breslauer Strasse 42 82194 Gröbenzell

Tel.: $081424484-80$ Fax: 08142 44848-20 www.blum-akupunktur.de info@blum-akupunktur.de

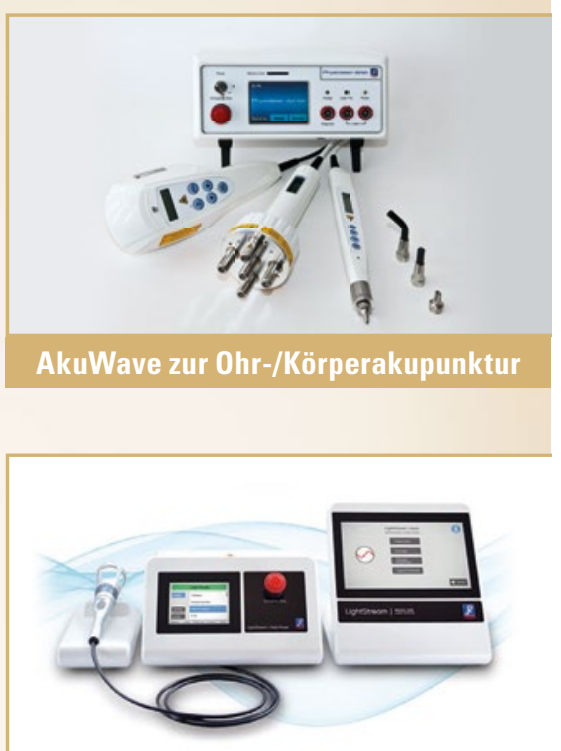

LightStream KI. 4 - Fläche und Tiefe

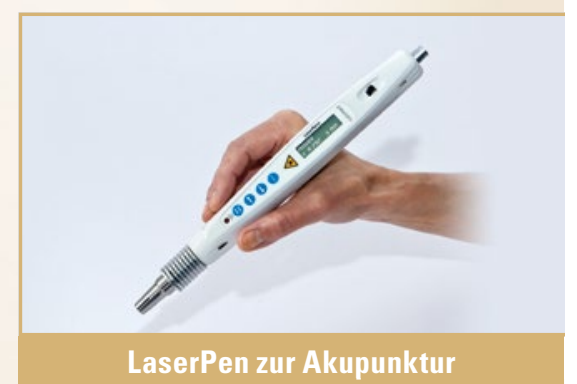

